\title{
A new physically based impact model for debris flow
}

\author{
XINGYUE LI* JIDONG ZHAO† and KENICHI SOGA
}

\begin{abstract}
A new analytical model is proposed to estimate the peak impact pressure of debris flow exerted on a rigid barrier. The new model consists of two terms, $p=\alpha_{1} \rho_{0} g h_{0}+0 \cdot 5 \rho_{0} u_{0}^{2}$, accounting for static and dynamic impacting effects, respectively. The static coefficient $\alpha_{1}$ is determined according to equations governing the mass and momentum conservation and energy conservation, and the dynamic coefficient 0.5 is adopted on the basis of the Bernoulli equation. The new analytical model is validated for a wide range of Froude number $(\mathrm{Fr})$ with data collected from past studies on small-scale experiments and field observations and numerical simulations of debris flow as a particle-fluid mixture performed by coupled computational fluid dynamics-discrete-element method (CFD-DEM, for wide-range coverage of Fr). Based on equivalence with the new model, the empirical coefficients involved in conventional pure hydrostatic $(k)$ and pure hydrodynamic $(\alpha)$ impact models are found positively and negatively correlated to Fr, respectively. A unique relationship between $k$ and $\alpha$ is further derived: $(\cos \theta / k)+(1 / \alpha)=1$, where $\theta$ denotes slope angle. The underlying physics of this relationship is interpreted. According to the proposed model, a design chart in terms of $\mathrm{Fr}$ is further recommended for practical design of debrisresisting barriers. The new analytical model offers a possible improvement on robust and reliable estimation of debris flow impact on a rigid barrier.
\end{abstract}

KEYWORDS: discrete-element modelling; landslides; numerical modelling; theoretical analysis; water flow

\section{INTRODUCTION}

Debris flows remain one of the most destructive natural hazards, causing great annual human fatalities and property losses across the world. Design and construction of countermeasures, such as rigid and flexible barriers, rely crucially on estimation of the impact of a debris flow, particularly its peak impact pressure, exerted on a debrisresisting structure. Popular analytical models proposed for predicting the peak impact pressure include the pure hydrostatic and hydrodynamic models. The hydrostatic model estimates the peak impact pressure $p$ based on flow density $\rho_{0}$ and flow height $h_{0}$ in conjunction with an empirical coefficient $k$ according to $p=k \rho_{0} g h_{0}$ (Lichtenhahn, 1973; Armanini, 1997). The hydrodynamic model calculates the peak impact pressure $p$ by the flow density $\rho_{0}$ and flow velocity $u_{0}$ with an empirical coefficient $\alpha$, according to $p=\alpha \rho_{0} u_{0}^{2}$ (Hungr et al., 1984; Scheidl et al., 2013). There are also hybrid models with combined consideration of hydrostatic and hydrodynamic factors, by either summing or multiplying the effects of flow height and flow velocity (Kherkheulidze, 1969; Arattano \& Franzi, 2003; Hübl et al., 2003). In addition to hydrostatic and hydrodynamic forces, other components such as drag force have also been considered in some studies (Vagnon \& Segalini, 2016).

These hydraulic models feature simple expressions, easy inputs of pertinent variables such as basic flow characteristics

Manuscript received 27 December 2018; revised manuscript accepted 22 April 2020

Discussion on this paper is welcomed by the editor.

* Department of Civil and Environmental Engineering, Hong Kong University of Science and Technology, Clearwater Bay, Kowloon, Hong Kong.

$\dagger$ Department of Civil and Environmental Engineering, Hong Kong University of Science and Technology, Clearwater Bay, Kowloon, Hong Kong (Orcid:0000-0002-6344-638X).

t Department of Civil and Environmental Engineering, University of California, Berkeley, Berkeley, CA, USA. (i.e. flow density, flow height and flow velocity) and convenience for calculation. They have been widely used in practical design of various debris-resisting barriers (including flexible barriers). However, major uncertainties that affect the accuracy of their predictions rest with the selection of their empirical coefficients (e.g. $k$ and $\alpha$ ). Frequently, the selection of their values lacks rigorous scientific guidelines for estimation (Cui et al., 2015), and their reported values vary substantially in the literature, depending on both the specific debris flow properties and the channel geometries (Cui et al., 2015; Vagnon \& Segalini, 2016). As discussed by Armanini et al. (2011) and Song et al. (2017), the determination of these empirical coefficients commonly involves considerable arbitrariness and subjective judgements and experience, affecting their consistency and accuracy for practical engineering design. Moreover, most models have been validated and fitted by data from limited cases of debris flows only, and their applicability for cases beyond the fitting data range remains to be proved.

Herein the authors present a new analytical study to predict the peak impact pressure of debris flow on a rigid barrier. In the derivation, the physical nature and fundamental governing equations are considered in describing a typical impact process of a debris flow on a barrier. The proposed analytical model will be validated by data encompassing a wide range of distinct flow regimes, including those collected from existing studies of small-scale experiments and real-scale observations on debris flows, as well as new coupled computational fluid dynamics-discrete-element method (CFD-DEM) simulations performed by the authors.

\section{A NEW ANALYTICAL MODEL FOR DEBRIS FLOW IMPACT}

In past studies, the peak impact pressure is commonly defined as the maximum impact pressure exerted on a barrier during the impact process (Hübl et al., 2009; Scheidl et al., 2013). The determination of peak impact pressure, however, can be complicated, as it may be both spatially and 
temporally dependent. Existing data obtained from both experiments and numerical simulations (Hübl et al., 2003; Armanini et al., 2011; Viccione et al., 2015; Song et al., 2017) indicate that the bottom of a rigid barrier may more often sustain the maximum impact and thus peak impact pressure. Experimental data (Cui et al., 2015) also show that the peak impact pressure is likely to be attained when the flow height in front of the barrier reaches its highest. Consequently, it is reasonable to assume that the worst-case scenario refers to the occurrence of peak impact pressure at the bottom of a barrier with a maximum run-up height of flow in front of the barrier (as shown in Fig. 1). The following analysis is based on a common no overflow case (e.g. the barrier is assumed to be sufficiently high) (Choi et al., 2015).

Referring to Fig. 1, the peak impact pressure is assumed to occur at the bottom of the barrier (e.g. point A). It consists of a hydrostatic part contributed from the run-up and a dynamic part caused by the dynamic impact of incoming flow according to the following expression

$$
p=p_{\mathrm{s}}+0 \cdot 5 \rho_{0} u_{0}^{2}
$$

where $p_{\mathrm{s}}$ is the static pressure. If the maximum run-up height is assumed to be $h_{1}$ and the static pressure is assumed to follow a hydrostatic pressure distribution, the peak impact pressure can be expressed as

$$
p=\rho_{0} g h_{1} \cos \theta+0 \cdot 5 \rho_{0} u_{0}^{2}
$$

where $\theta$ is the slope angle. The dynamic impact part $0 \cdot 5 \rho_{0} u_{0}^{2}$ has been considered on the basis of the Bernoulli equation (Erhard et al., 2010), where $\rho_{0}$ and $u_{0}$ are the density and velocity of incoming flow, respectively. Herein it is assumed that the incoming flow with velocity $u_{0}$ only changes its flow direction upon the impact, while no energy is lost. Indeed, experimental analysis of flow trajectory in a flow clearly demonstrates that the flow near the bottom of the barrier is dominantly moving upward when reaching the maximum run-up height (Armanini et al., 2011).

A notable difference of the above new proposal, as compared to previous combined type impact models (e.g. Kherkheulidze, 1969; Arattano \& Franzi, 2003; Hübl et al., 2003 ), is the consideration of the static load from the accumulated flow climbing on the barrier, in addition to the dynamic impact of the incoming flow from the slope. If the maximum run-up height $h_{1}$ is further related to the height of the incoming flow $h_{0}$, the peak impact pressure in equation (2) can be recast into

$$
p=\alpha_{1} \rho_{0} \boldsymbol{g} h_{0}+0 \cdot 5 \rho_{0} u_{0}^{2}
$$

where $\alpha_{1}$ is a static coefficient

$$
\alpha_{1}=\frac{h_{1} \cos \theta}{h_{0}}
$$

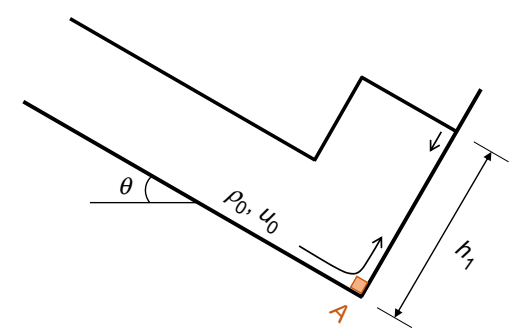

Fig. 1. Flow condition of peak impact pressure and maximum run-up considered in the new impact model. $h_{1}$ is the maximum run-up height on the barrier. $\theta$ is slope angle. $\rho_{0}$ and $u_{0}$ are the density and velocity of the incoming flow, respectively $\alpha_{1}$ is introduced here to reflect the ratio of the maximum run-up height to the height of incoming flow. Its physical justification and determination in accounting for different flow regimes and flow conditions are detailed in the following.

When the slope inclination $\theta$ is known and the incoming flow height $h_{0}$ for a design site/flow is provided or estimated from the historical data (Lo, 2000), $h_{1} / h_{0}$ must be determined to calculate $\alpha_{1}$. In determining $h_{1} / h_{0}$ and therefore $\alpha_{1}$, two different approaches are considered here. One is based on the conservation of mass and momentum (Jóhannesson et al., 2009) and the other on energy conservation (Kwan, 2012). A conservative solution between the two for different cases of Froude number is then suggested. Note that both approaches have been based on an assumption of a steady, uniform, homogeneous and one-dimensional incoming flow (Armanini et al., 2011; Iverson et al., 2016).

\section{$\mathrm{h}_{1} / \mathrm{h}_{0}$ based on mass and momentum conservation}

Mass and momentum conservation laws have been widely used for estimating the maximum run-up height (Armanini, 1997; Jóhannesson et al., 2009; Armanini et al., 2011; Iverson et al., 2016). They are employed here to describe the case of debris flow shown in Fig. 2. The debris mass shown in the dashed rectangle in the figure is selected as the control volume for analysis

$$
\left\{\begin{array}{l}
\rho_{0} h_{0}\left(u_{0}+u_{1}\right)=\rho_{1} h_{1} u_{1} \\
\frac{1}{2} \kappa\left(\rho_{0} h_{0}^{2}-\rho_{1} h_{1}^{2}\right) \boldsymbol{g} \cos \theta=\rho_{1} h_{1} u_{1}^{2}-\rho_{0} h_{0}\left(u_{0}+u_{1}\right)^{2}
\end{array}\right.
$$

where $\rho_{0}$ and $\rho_{1}$ are the densities of the incoming flow and the reflected flow at impact, respectively, and $h_{0}$ and $h_{1}$ are their respective heights. $u_{0}$ and $u_{1}$ are the velocities of the incoming flow and the reflected flow, respectively. $\kappa$ is a pressure coefficient accounting for converging and diverging flow modes of debris flow. It is defined as the ratio between the tangential stress and normal stress in the flowing mass $\left(\sigma_{3}\right.$ and $\sigma_{1}$ in Fig. 2), which is equivalent to Rankine's earth pressure coefficient (Scheidl et al., 2014). The pressure coefficient $\kappa$ was originally proposed for a dry, granular flow and later extended for debris flows (Hungr, 1995; Iverson \& Denlinger, 2001; Iverson et al., 2010, 2016). For regions of converging flow with a compressional deformation mode, the normal stress is smaller than the tangential stress, so $\kappa>1$ (i.e. passive earth pressure coefficient). In regions of diverging flow with an extensional deformation mode, $\kappa<1$ (i.e. active earth

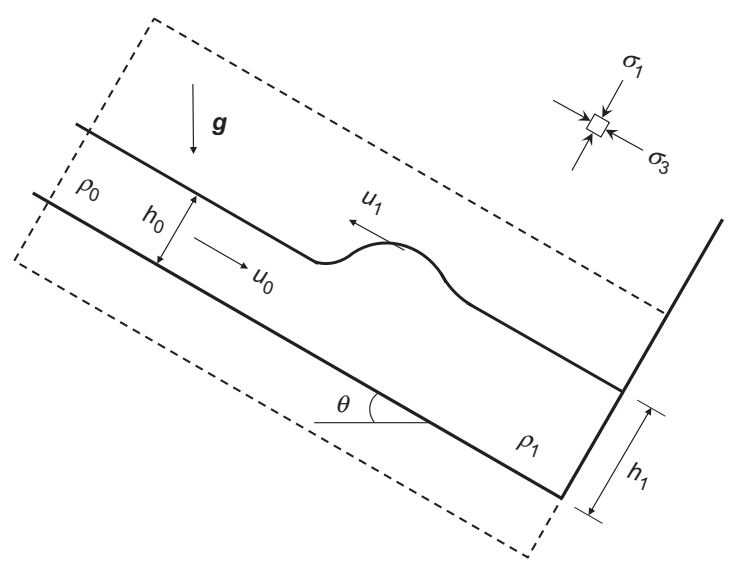

Fig. 2. Schematic representation of the determination of $h_{1} / h_{0}$ based on mass and momentum conservation. The debris mass in the dashed rectangle is selected as the control volume 
pressure coefficient). For a typical dry debris, the value of the pressure coefficient is between $0 \cdot 2$ and $5 \cdot 0$ (Hungr, 1995).

The governing mass and momentum equations can be recast into the following single expression (see the derivation in Appendix 1)

$$
\beta\left(\frac{h_{1}}{h_{0}}\right)^{3}-\left(\frac{h_{1}}{h_{0}}\right)^{2}-\left(1+\frac{2 \mathrm{Fr}^{2}}{\kappa}\right)\left(\frac{h_{1}}{h_{0}}\right)+\frac{1}{\beta}=0
$$

where $\beta$ is the density ratio $\rho_{1} / \rho_{0}$; Fr is Froude number defined as the ratio between flow inertia and external field (gravity here). For a flow over an inclined slope of angle $\theta, \mathrm{Fr}$ is defined as follows

$$
\operatorname{Fr}=u_{0} / \sqrt{g h_{0} \cos \theta}
$$

Based on Cardano's solution of a cubic equation (Cardano, 1545), $h_{1} / h_{0}$ can be solved as follows

$$
\frac{h_{1}}{h_{0}}=2 \sqrt[3]{r} \cos \zeta+\frac{1}{3 \beta}
$$

where

$$
\begin{aligned}
& r=\sqrt{-\left(\frac{e}{3}\right)^{3}} \\
& e=\frac{-3 \beta\left(1+2 \mathrm{Fr}^{2} / \kappa\right)-1}{3 \beta^{2}} \\
& \zeta=\frac{1}{3} \arccos \left(\frac{-q}{2 r}\right) \\
& q=\frac{27 \beta-9 \beta\left(1+2 \mathrm{Fr}^{2} / \kappa\right)-2}{27 \beta^{3}}
\end{aligned}
$$

Consequently, the analytical solution for $h_{1} / h_{0}$ in equation (8) is a function of Froude number, Fr, pressure coefficient, $\kappa$, and density ratio, $\beta$.

\section{$\mathrm{h}_{I} / \mathrm{h}_{0}$ based on energy conservation}

Energy conservation has been used for estimating the maximum run-up height (Armanini et al., 2011). Consider a flow point with mass $m$ (the shaded squares shown in Fig. 3) at the free surface immediately prior to the impact. Assume its kinetic energy will all be transformed into potential energy without energy loss after it impacts and climbs up the barrier to a maximum run-up height. In this process, the following energy transformation holds

$$
\frac{1}{2} m u_{0}^{2}=m \boldsymbol{g}\left(h_{1}-h_{0}\right) \cos \theta
$$

The ratio of the maximum run-up height to the height of the incoming flow can be readily obtained

$$
\frac{h_{1}}{h_{0}}=1+0 \cdot 5 \mathrm{Fr}^{2}
$$

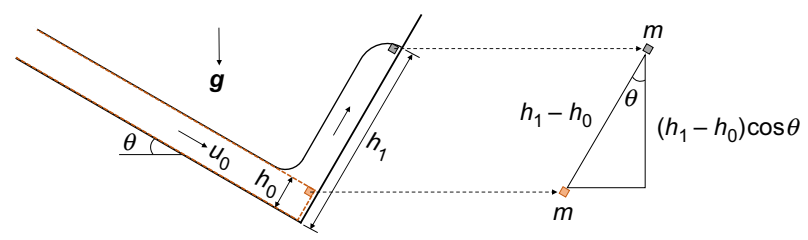

Fig. 3. Schematic representation of the determination of $h_{1} / h_{0}$ based on energy conservation

\section{Conservative selection between the two solutions}

As different flow schemes are assumed in the above two approaches, they may be adopted for flows with distinct characteristics and regimes. The approach of mass and momentum conservation does not guarantee conservation of mechanical energy, and hence potentially underestimates the run-up height according to the experimental data by Choi et al. (2015). The approach of energy conservation assumes zero energy loss, which may be too conservative for flows with notable energy dissipation (e.g. the frictional flows in Choi et al. (2015)). Indeed, it was reported by Choi et al. (2015) that the mass and momentum conservation approach gives a more accurate prediction on dry sand, whereas the energy conservation approach performs better for water. Iverson et al. (2016) suggested use of mass and momentum conservation for saturated debris flows. Nevertheless, Armanini et al. (2011) claimed that both flow schemes in Figs 2 and 3 may occur for a pure fluid and for a two-phase flow composed of particles and a liquid. As there are no measured data of the density ratio $\beta$ and the pressure coefficient $\kappa$ for saturated debris flows, the justification on the applicability of the two approaches has been rather limited. A conservative solution is therefore suggested here for safety reasons.

The above two solutions may provide apparently different predictions on the ratio of the maximum run-up height and the height of incoming flow $h_{1} / h_{0}$, as presented in Figs 4 and 5. The plotted Fr from 0 to 12 refers to debris flows in experiments and real cases (Hübl et al., 2009). The pressure coefficient $\kappa$ in Fig. 4 ranges from $0 \cdot 2$ to $5 \cdot 0$, which is typical for granular flows (Hungr, 1995; Iverson et al., 2016). Referring to the experimental measurements of dry, granular flows by $\mathrm{Ng}$ et al. (2017), a density ratio $\beta$ from $1 \cdot 0$ to $2 \cdot 0$ has been taken into account in Fig. 5. Note theoretical predictions with a density ratio from 1.0 to 3.0 are presented in $\mathrm{Ng}$ et al. (2017). Nevertheless, as no experimental data fall on the theoretical curve with a density ratio of $3 \cdot 0$, and it is difficult to have a high density ratio of 3.0 in the saturated and uniform granular flows assumed in this study, $\beta$ up to $2 \cdot 0$ has been considered in Fig. 5. In addition, $\beta$ smaller than 1 is included in Fig. 5 according to the current authors' simulation result with $\beta<1$ (see details in the validation part).

For the special case where both $\beta$ and $\kappa$ are 1, as considered for fluid by Armanini et al. (2011) and Choi et al. (2015), it is interesting to observe that the two solutions indeed have an intersection point at $\mathrm{Fr}=2 \cdot 54$. When $\mathrm{Fr}<2 \cdot 54$, the

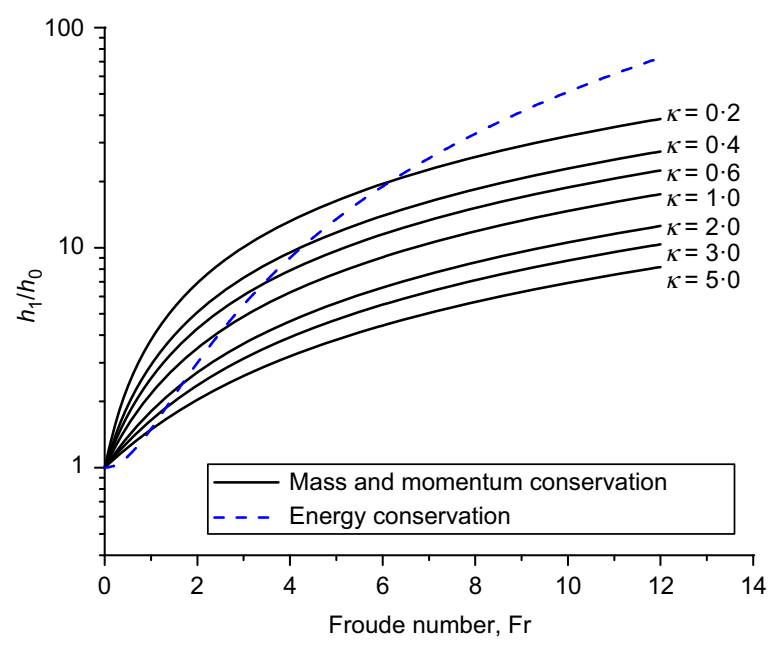

Fig. 4. Solutions from two sets of governing equations: mass and momentum conservation (density ratio $\beta=1$, pressure coefficient $\mathbf{0 \cdot 2} \leq \boldsymbol{\kappa} \leq \mathbf{5 \cdot 0}$ ) as well as energy conservation 


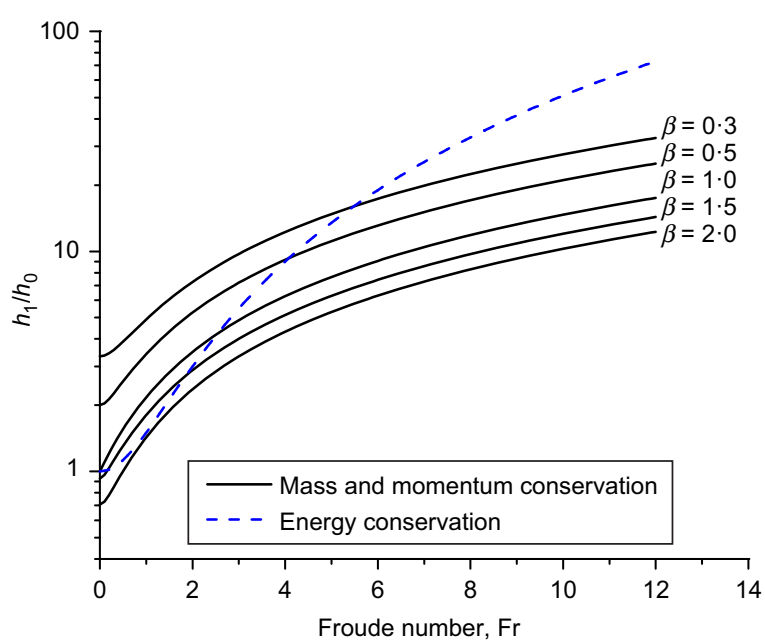

Fig. 5. Solutions from two sets of governing equations: mass and momentum conservation (pressure coefficient $\kappa=1$, density ratio $\mathbf{0 \cdot 3} \leq \boldsymbol{\beta} \leq \mathbf{2 \cdot 0}$ ) as well as energy conservation

mass/momentum conservation solution proves to be more conservative than the energy conservation solution, and the latter becomes more conservative when $\mathrm{Fr}>2 \cdot 54$. This observation is consistent with that presented by Choi et al. (2015). Moreover, the present study clearly shows that the intersection point may indeed depend on both the pressure coefficient $\kappa$ and density ratio $\beta$. At lower $\kappa$ or $\beta$, the intersection may occur at relatively higher Fr. Notably from Fig. 5, for the case with $\kappa=1$ and $\beta=2 \cdot 0$, the energy solution is more conservative for the whole range of Fr because there is no intersection at all. Note also that the case of $\beta=1.5$ has two intersection points between the two solutions, which may slightly complicate practical selection of a more conservative solution between the two.

In summary, the following procedures are suggested for the proposed analytical formula equation (3) to predict the peak impact pressure.

(a) Obtain the velocity $u_{0}$, height $h_{0}$, density $\rho_{0}$ of the incoming flow and the slope angle $\theta$. Given a known design flow/site, the slope angle $\theta$ can be estimated. The other three parameters can be determined from historical data considering the source material of the design site, the estimated magnitude (volume) of debris flow, the super-elevation and the channel property (Lo, 2000; Rahman \& Konagai, 2017).

(b) Estimate the density ratio, $\beta$, and pressure coefficient, $\kappa$, based on existing data from laboratory experiments and real-scale measurements as well as numerical simulations (such as CFD-DEM presented below).

(c) Obtain the ratio of maximum run-up height and height of incoming flow $h_{1} / h_{0}$ based on mass and momentum conservation. With a flow velocity $u_{0}$, flow height $h_{0}$ and slope angle $\theta$, the Froude number Fr can be calculated according to equation (7). The $h_{1} / h_{0}$ can be obtained based on equations (8)-(12) by plugging in the $\mathrm{Fr}, \beta$ and $\kappa$.

(d) Calculate the ratio of maximum run-up height and height of incoming flow $h_{1} / h_{0}$ based on energy conservation from equation (14). The Froude number in equation (14) is calculated in the same way as shown in step $(c)$.

(e) Compare the values of $h_{1} / h_{0}$ acquired from steps (c) and $(d)$, choose the larger one to calculate $\alpha_{1}$ based on equation (4).
( $f$ ) Calculate the peak impact pressure with equation (3) by plugging in the static coefficient $\alpha_{1}$, flow density $\rho_{0}$, flow height $h_{0}$ and flow velocity $u_{0}$.

Linking between conventional pure hydraulic models

The newly proposed model can indeed help to establish a linking between conventional pure hydraulic models. Recall the pure hydrostatic model and pure hydrodynamic model in their definitions of empirical coefficients

$$
\begin{aligned}
& k=\frac{p}{\rho_{0} \boldsymbol{g} h_{0}} \\
& \alpha=\frac{p}{\rho_{0} u_{0}^{2}}
\end{aligned}
$$

where $k$ and $\alpha$ are the empirical hydrostatic and hydrodynamic coefficients in conventional hydraulic models (Lichtenhahn, 1973; Hungr et al., 1984; Scheidl et al., 2013). If the peak pressure $p$ is assumed to be identical in all the three models, the following relationships can be established between the two conventional hydraulic models and the authors' new model in equation (3)

$$
\begin{aligned}
& k=\frac{p}{\rho_{0} g h_{0}}=\alpha_{1}+0 \cdot 5 \mathrm{Fr}^{2} \cos \theta \\
& \alpha=\frac{p}{\rho_{0} u_{0}^{2}}=\frac{\alpha_{1}}{\cos \theta} \mathrm{Fr}^{-2}+0 \cdot 5
\end{aligned}
$$

By eliminating Fr in equations (17) and (18), the following relationship can be found among the three coefficients, $\alpha_{1}$ in the present model, $k$ in the hydrostatic model and the $\alpha$ in the hydrodynamic model

$$
\frac{\alpha_{1}}{k}+\frac{0 \cdot 5}{\alpha}=1
$$

As demonstrated in Appendix 2, if energy conservation is considered, substitution of equation (14) in equation (4) leads to

$$
\alpha_{1}=\left(1+0 \cdot 5 \mathrm{Fr}^{2}\right) \cos \theta
$$

If equation (20) is substituted into equations (17) and (18), the conventional hydraulic impact model coefficients have the following expressions in terms of $\mathrm{Fr}$

$$
\begin{aligned}
& k=\left(1+\mathrm{Fr}^{2}\right) \cos \theta \\
& \alpha=1+\mathrm{Fr}^{-2}
\end{aligned}
$$

From equations (21) and (22), $k$ has a positive correlation with Fr, whereas $\alpha$ is negatively correlated with Fr. Similar correlations have been observed in previous studies (Hübl et al., 2009; Proske et al., 2011; Scheidl et al., 2013), which will be further verified by the present authors' numerical simulation in the next section.

Indeed, based on energy conservation, the following reciprocal relationship can be further established between the two conventional impact coefficients $k$ and $\alpha$ if one combines equations (7), (15), (16), (19) and (20)

$$
\frac{\cos \theta}{k}+\frac{1}{\alpha}=1
$$

Further involvement of Fr leads to the following equation by combining equations (21) and (22)

$$
\mathrm{Fr}^{2}=\frac{k}{\alpha} \frac{1}{\cos \theta}
$$




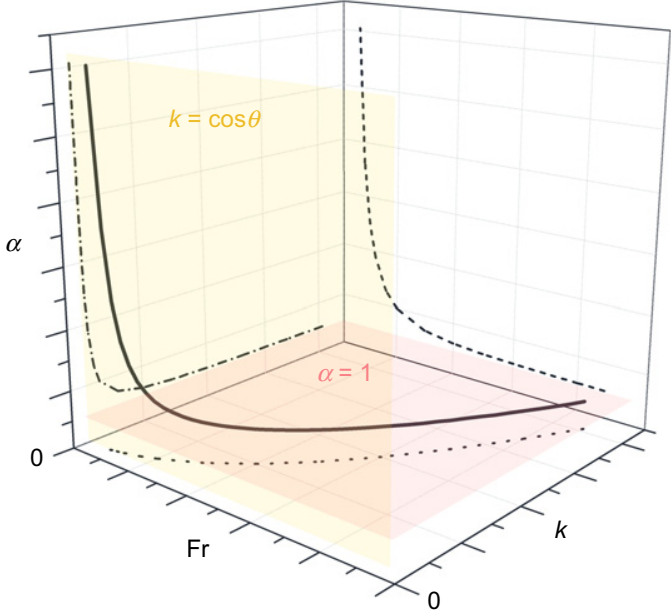

Fig. 6. Relation of hydrostatic coefficient $k$, hydrodynamic coefficient $\alpha$ and Froude number Fr. Assuming a horizontal channel $\left(\theta=0^{\circ}\right)$, the projected curves on $k-F r$ and $\alpha-F r$ planes follow equations (21) and (22), respectively. The projected curve on the $\alpha-k$ plane satisfies equation (23). Faces parallel to the $\alpha-\mathrm{Fr}$ and $k-\mathrm{Fr}$ planes are $k=\cos \theta$ and $\alpha=1$, respectively

While equation (23) explicitly exhibits an inversely proportional relation between $k$ and $\alpha$, equation (24) presents itself as a spatially unique curve in the space of $\mathrm{Fr}-\alpha-k$, which can be illustrated as shown in Fig. 6 . The projection of this curve onto the $k-\mathrm{Fr}$ plane is equation (21), and its projection onto the $\alpha-$ Fr plane is equation (22). The projection of this curve onto the $\alpha-k$ plane depicts clearly the inversely proportional relationship between $k$ and $\alpha$ expressed by equation (23). Given a real flow with velocity $u_{0} \geq 0$ and flow height $h_{0}>0$, the general ranges for $k$ and $\alpha$ are: $k \geq \cos \theta$ and $\alpha>1$.

If the mass and momentum conservation is considered, a different, relatively more complicated form of dependence between $k$ and $\alpha$ can be found, involving terms such as pressure coefficient $\kappa$, density ratio $\beta$ and slope angle $\theta$. This is briefly discussed in Appendix 2.

\section{VALIDATION OF THE NEW IMPACT MODEL BY CFD-DEM SIMULATIONS, FIELD AND LABORATORY TESTING DATA}

The density ratio $\beta$ and pressure coefficient $\kappa$ are difficult to measure from experiments and real debris flows. To validate the analytical model, numerical simulations have been conducted based on a coupled CFD-DEM approach. All quantities required for the analytical model can be readily extracted from the CFD-DEM simulations for comparison, and the numerical simulations can also be easily conducted to cover a wide range of flow regimes observed in both small-scale experiments and real debris flows. Validation of the proposed analytical model by available field observations and laboratory testing data will be further presented.

\section{Coupled CFD-DEM of debris flow impacts}

A coupled CFD-DEM approach enables realistic modelling of debris flow where fluid-particle interactions dictate its dynamics and impacts (Li \& Zhao, 2018a, 2018b). In the coupled CFD-DEM approach, the CFD (Anderson \& Jackson, 1967) is used to solve the locally averaged Navier-Stokes equations for the fluid and the DEM (Tsuji et al., 1992, 1993) is used to solve the Newton's equation governing a granular particle system. Two open source software packages, namely, OpenFoam (Weller et al., 1998) and LIGGGHTS (Kloss \& Goniva, 2010), are employed for the CFD and DEM engines, respectively. The coupling between the CFD and DEM is realised by exchanging interaction forces including drag force, buoyancy and viscous force, based on an interface program CFDEM originally developed by Goniva et al. (2010) and later extended by Zhao \& Shan (2013). Details of the numerical schemes can be found in Zhao \& Shan (2013) and Shan \& Zhao (2014) and will not be repeated here.

Model set-up for simulation. To examine the analytical solution under different flow conditions, three groups of coupled CFD-DEM simulations on debris flow impact are conducted as summarised in Table 1. The model set-ups of the simulations are illustrated in Fig. 7. Group I and group II share the set-up in Fig. 7(a), and group III uses the set-up in Fig. 7(b). For all the simulations, a rigid barrier is placed over a horizontal or inclined channel with a length, $L_{\mathrm{c}}$. An inlet with a height $h$ is placed at the left side of the channel to supply a source of debris mixture, where the debris mixture flows out of the inlet into the channel at a prescribed initial velocity, $u$. For both group I and group II in Fig. 7(a), a prismatic debris sample with side length of $l$, flow height $h$ and flow velocity $u$ is initially placed on top of the slope, where the flow height and flow velocity are consistent with the flow from the inlet. All the debris mass in group III comes from the inlet without an initial prismatic sample (Fig. 7(b)). Initial debris samples with a velocity from $0 \cdot 2$ to $8.25 \mathrm{~m} / \mathrm{s}$ are adopted in groups I and II to obtain flows with relatively small Froude numbers. In group III, the inlet injects flows of an initial velocity from $14 \cdot 0$ to $22.0 \mathrm{~m} / \mathrm{s}$ to produce flows with large Froude numbers. Note that it is challenging to obtain flows with small Froude numbers by using injecting flows with very small velocity from the inlet, since the flow height decreases during the propagation along the channel. Therefore, the initial sample is placed close to the barrier in groups I and II where the propagation phase is disregarded (Ceccato et al., 2017). In addition, both the fluid and solid phases of a debris mixture are varied to reproduce debris flows with distinct constituents. The initial solid fractions of the debris mixture are $63.36 \%$, $42.53 \%$ and $36.91 \%$ in group I, group II and group III, respectively, in reference to real debris flows (Coussot \& Meunier, 1996; Takahashi, 2014). Bi-disperse particles are used for debris flows in all three groups to save computational cost. The fraction of the large $\left(d_{\mathrm{p}}=0.02 \mathrm{~m}\right)$ and small $\left(d_{\mathrm{p}}=0.012 \mathrm{~m}\right)$ particles in the granular system are $70 \%$ and $30 \%$ for groups I and II, and $50 \%$ and $50 \%$ for group III, respectively. Water is considered as the debris fluid in groups I and II. However, the use of water in group III may possibly induce inhomogeneous flows due to high velocity; a mud flow with higher density and viscosity is adopted (Huang \& Garcia, 1998) by using a Herschel-Bulkley fluid model. The contact parameters for debris particles refer to Byerlee (1978), Zhao \& Shan (2013) and Hurley et al. (2016). Detailed parameters employed in the simulations are summarised in Table 1.

\section{Comparison between analytical predictions with numerical simulations}

Relevant parameters required for the analytical model, including $u_{0}, h_{0}, \rho_{0}, \theta, \beta$ and $\kappa$, are extracted from the CFD-DEM results for calculating the peak impact pressure, which is further compared against the simulated values. A representative case in group III is presented in Fig. 8 to illustrate the extraction of relevant parameters for comparison. Before the simulated debris mixture impacts on the barrier, the velocity and height of the incoming flow (i.e. $u_{0}$ and $h_{0}$ ) are 
Table 1. Selected parameters for the three groups of coupled CFD-DEM simulations

\begin{tabular}{|c|c|c|c|}
\hline & Group I & Group II & Group III \\
\hline \multicolumn{4}{|l|}{ Geometry } \\
\hline$L_{\mathrm{c}}: \mathrm{m}$ & 2 & 2 & 3 \\
\hline$l: \mathrm{m}$ & 1.9 & 1.9 & 0 \\
\hline$h: \mathrm{m}$ & $0 \cdot 4$ & $0 \cdot 4$ & $0 \cdot 4$ \\
\hline$\theta:$ degrees & 0 & 0 & 35 \\
\hline \multicolumn{4}{|l|}{ Initial velocity } \\
\hline$u: \mathrm{m} / \mathrm{s}$ & $0 \cdot 2-2 \cdot 65$ & $3 \cdot 0-8 \cdot 25$ & $14 \cdot 0-22 \cdot 0$ \\
\hline \multicolumn{4}{|l|}{ Debris fluid } \\
\hline Density: $\mathrm{kg} / \mathrm{m}^{3}$ & 1000 & 1000 & 1235 \\
\hline Consistency, $\kappa_{\mathrm{c}}: \mathrm{Pa} \mathrm{s}{ }^{n}$ & $0 \cdot 001$ & $0 \cdot 001$ & $1 \cdot 7$ \\
\hline Flow index, $n$ & 1 & 1 & $0 \cdot 36$ \\
\hline Yield stress, $\tau_{c}: \mathrm{Pa}$ & 0 & 0 & $2 \cdot 91$ \\
\hline \multicolumn{4}{|l|}{ Debris particle } \\
\hline Diameter, $d_{\mathrm{p}}: \mathrm{m}$ & $0 \cdot 02,0 \cdot 012$ & $0 \cdot 02,0 \cdot 012$ & $0 \cdot 02,0 \cdot 012$ \\
\hline Density: $\mathrm{kg} / \mathrm{m}^{3}$ & 2500 & 2500 & 2500 \\
\hline Young's modulus: GPa & 70 (particle-particle) & 70 (particle-particle) & 70 (particle-particle) \\
\hline Young's modulus: GPa & 700 (particle-wall) & 700 (particle-wall) & 700 (particle-wall) \\
\hline Poisson's ratio & $0 \cdot 3$ & $0 \cdot 3$ & $0 \cdot 3$ \\
\hline Restitution coefficient & $0 \cdot 7$ & 0.7 & $0 \cdot 7$ \\
\hline Inter-particle friction coefficient & $0 \cdot 7$ & $0 \cdot 7$ & $0 \cdot 7$ \\
\hline Particle-wall friction coefficient & 0.7 & 0.7 & $0 \cdot 7$ \\
\hline Rolling friction coefficient & $0 \cdot 1$ & $0 \cdot 1$ & $0 \cdot 1$ \\
\hline \multicolumn{4}{|l|}{ Simulation control } \\
\hline DEM time step: s & $5 \times 10^{-7}$ & $5 \times 10^{-7}$ & $5 \times 10^{-7}$ \\
\hline CFD time step: $s$ & $5 \times 10^{-5}$ & $5 \times 10^{-5}$ & $5 \times 10^{-5}$ \\
\hline CFD cell size. $\mathrm{m}^{3}$ & $1 \times 1 \times 1$ & $1 \times 1 \times 1$ & $1 \times 1 \times 1$ \\
\hline CFD cell size: $\mathrm{m}$ & $\overline{30} \times \overline{30} \times \overline{30}$ & $\overline{30} \times \overline{30} \times \overline{30}$ & $\overline{30} \times \overline{30} \times \overline{30}$ \\
\hline
\end{tabular}

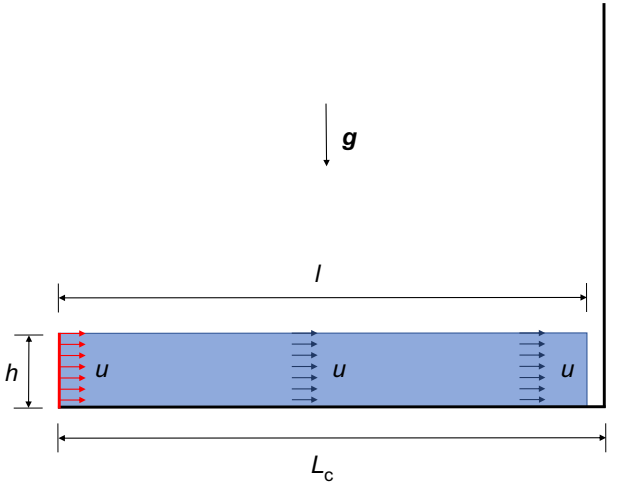

(a)

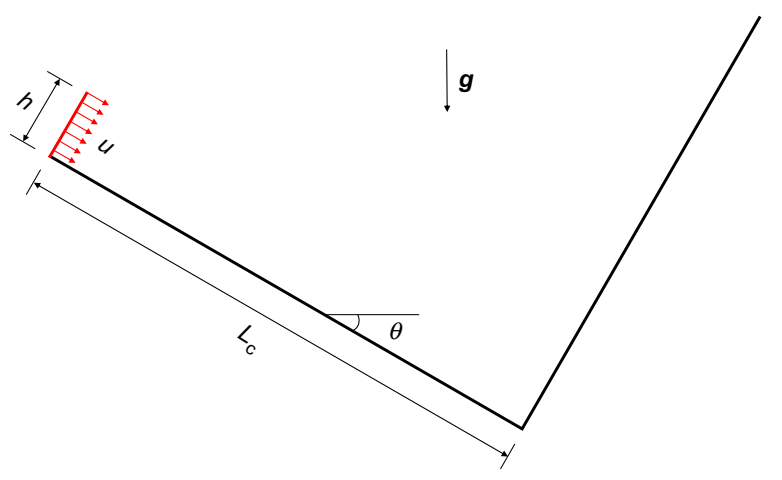

(b)

Fig. 7. Model set-ups of coupled CFD-DEM simulations for (a) groups I and II and (b) group III (the thickness of the three-dimensional model set-ups is $0.3 \mathrm{~m}$ )

determined within a section of debris mass (between the two dashed lines in Fig. 8). This section is selected to reflect both the front velocity (Hu et al., 2011; Scheidl et al., 2013) and the height of the main body. The size of section $0.1 \mathrm{~m}$ is

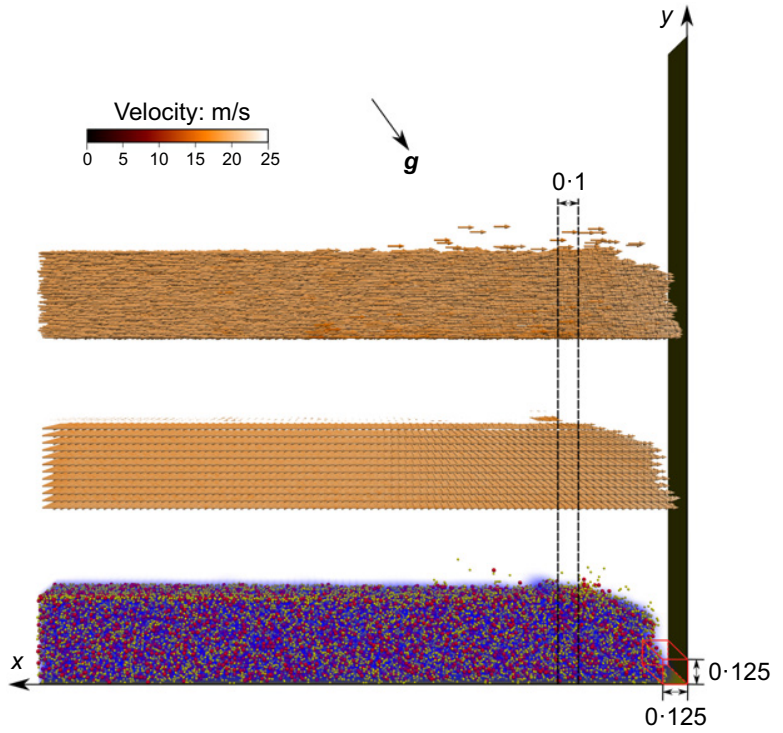

Fig. 8. A representative case (group III, $u=20 \mathrm{~m} / \mathrm{s}$ ) in determining the height and velocity of the incoming flow. The bottom panel shows the debris mixture before the impact, which is composed of a liquid, particles with a diameter of $0.02 \mathrm{~m}$, and particles with a diameter of $0.012 \mathrm{~m}$; the middle and upper panels show the velocity fields of the liquid phase and the particles, respectively. The two dashed lines define the selected section to determine parameters before the impact. The cuboid at the bottom of the barrier shows the position of debris mass used to calculate the parameters after the impact. The size of the cuboid is determined according to the space extracting the impact pressure. Unit of length: $m$

determined to have sufficient particles along the flow direction ( $\geq 5$ in this study) for obtaining representative data, and to include complete CFD cells for convenient data extraction from the CFD. Note the simulated flows are rather uniform as shown in Fig. 8, thus the extracted values do not have any 
notable change if the section is shifted to the left or the size of the section is increased by moving its left bound to the left. For debris flows in laboratory experiments (Hu et al., 2011), large-scale tests (Bugnion et al., 2012) and real-scale measurements $(\mathrm{Hu}$ et al., 2011), the front velocity is commonly suggested as the velocity of the incoming flow. The density of the incoming flow $\rho_{0}$ is determined with the selected section as well. The slope angle $\theta$ is known from the model set-up. To obtain the density ratio $\beta$ and the pressure coefficient $\kappa$, the density of the reflected flow $\rho_{1}$ as well as the tangential and normal stresses in the reflected flow $\left(\sigma_{3}\right.$ and $\left.\sigma_{1}\right)$ at the instant of peak impact pressure should be determined. Since the peak impact pressure occurs at the bottom of the barrier, as demonstrated in Fig. 9, a cuboid of debris mass at the bottom of the barrier (see Fig. 8) is used to calculate the density and the stresses of the reflected flow. The peak impact pressure is extracted from the bottom section of the barrier with dimensions $0.125 \mathrm{~m} \times 0.3 \mathrm{~m}$. Correspondingly, the size of the cuboid debris mass is determined as $0 \cdot 125 \times 0 \cdot 125 \times 0.3 \mathrm{~m}$. Note it is assumed that the cuboid debris mass is within the reflected flow and its properties and stresses are representative at the instant of peak impact pressure. After $\rho_{1}$ is obtained, the density ratio $\beta$ can be calculated as $\rho_{1} / \rho_{0}$. Referring to the directions of tangential and normal stresses in Fig. 2, the tangential stress of the cuboid debris mass $\sigma_{3}$ is backcalculated as the pressure on the bottom section of the barrier, while its normal stress $\sigma_{1}$ is back-calculated as the pressure on the section of the channel bed below the cuboid debris mass. The pressure coefficient $\kappa$ can then be readily obtained $\left(\sigma_{3} / \sigma_{1}\right)$.

To facilitate the following analyses and comparison, the peak impact pressure, either predicted by the analytical model in equation (3) or obtained from the numerical simulations, will be normalised by the static component $\rho_{0} g h_{0}$ and the dynamic component $\rho_{0} u_{0}^{2}$, respectively, according to equations (15) and (16). Consequently, the normalised quantities provide equivalences that are comparable to $k$ and $\alpha$ in conventional hydraulic models.

Figures 10 and 11 compare the variations of $k$ and $\alpha$ with Froude number Fr predicted by the analytical solutions and the simulation results. In total, 29 simulations have been conducted (eight in group I, 16 in group II and five in group III) for the data points in Figs 10 and 11, covering different flow regimes from subcritical flows $(\mathrm{Fr}<1)$ to supercritical flows $(\mathrm{Fr}>1)$. Notably, the range of Froude number reported in the literature for real observations and experiments is typically from 0 to 12 (Hübl et al., 2009). Three

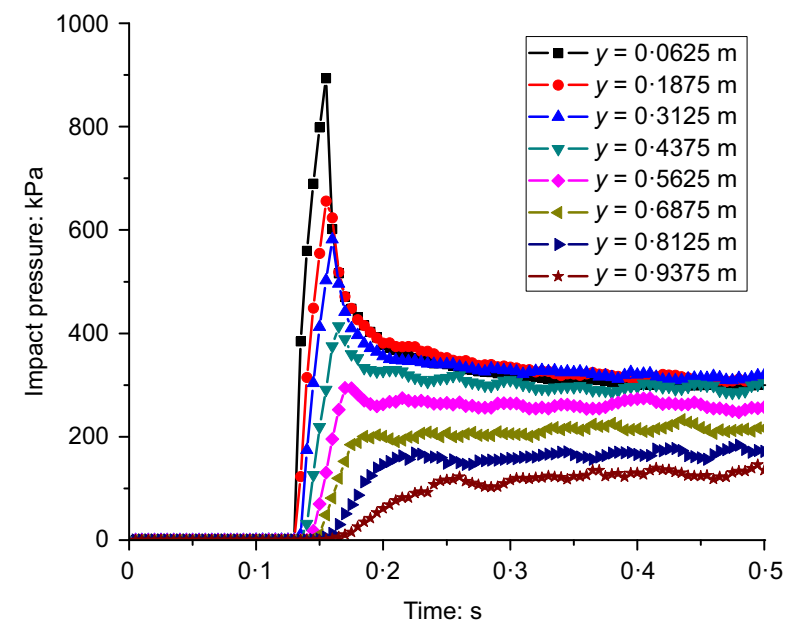

Fig. 9. Pressure distribution on the barrier in a representative case (group III, $u=20 \mathrm{~m} / \mathrm{s}$ )

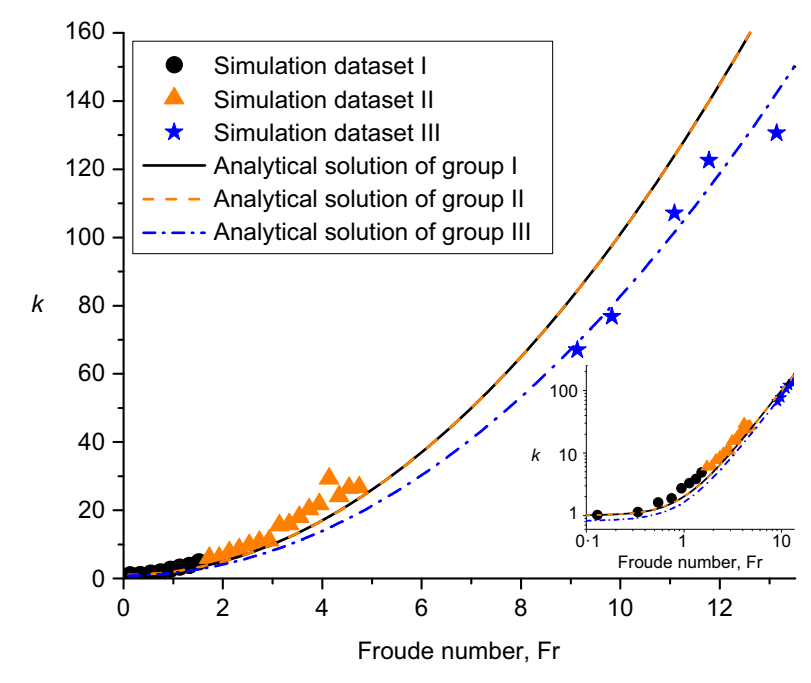

Fig. 10. Comparison of the simulation data and analytical predictions of the relation between the hydrostatic coefficient $k$ and Froude number Fr (shown inset on a double-log scale)

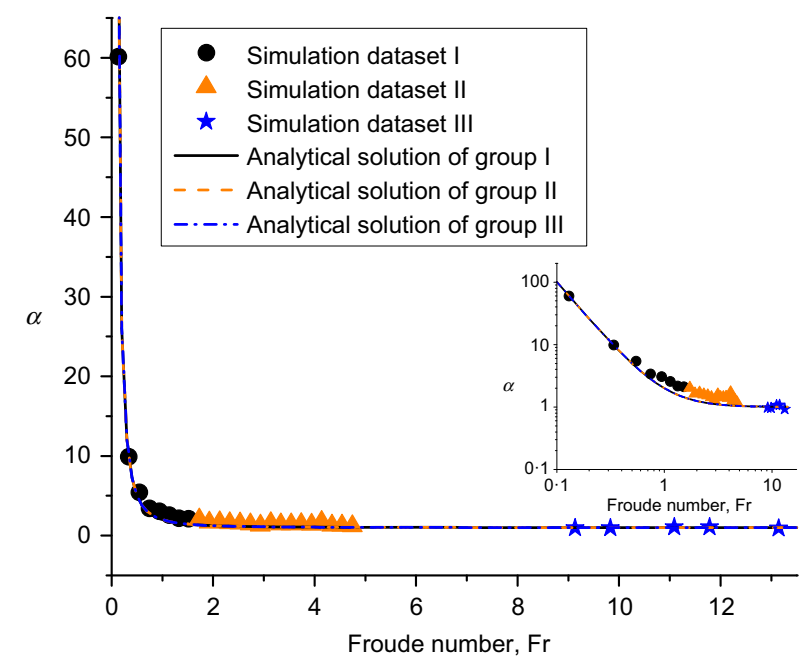

Fig. 11. Comparison of the simulation data and analytical predictions of the relation between the hydrodynamic coefficient $\alpha$ and Froude number Fr (shown inset on a double-log scale)

analytical solutions are demonstrated in both figures. Based on the extracted $\sigma_{1}, \sigma_{3}, \rho_{0}$ and $\rho_{1}$ from the simulations, the average pressure coefficients $\kappa$ in the three groups are calculated as $1 \cdot 895,1 \cdot 800$ and $1 \cdot 316$, and their average density ratios $\beta$ are $0.995,1.017$ and 1.016 , respectively. Having these average parameters, $h_{1} / h_{0}$ values derived from the energy conservation approach are more conservative compared to those from the mass and momentum conservation approach. Hence the three analytical solutions in both figures have been based on $h_{1} / h_{0}$ derived from energy conservation. At a given Froude number, the distinction in the three analytical solutions of $k$ in Fig. 10 stems from the slope angle $\theta$ (see equation (21)). A steeper slope results in a smaller empirical hydrostatic coefficient, $k$. This explains why the horizontal channels in groups I and II lead to identical solutions, while the steeper channel in group III results in a smaller $k$ in Fig. 10. As the empirical hydrodynamic coefficient $\alpha$ in equation (22) is exclusively dependent on Froude number, the analytical solutions for three simulation groups coincide entirely with one another in Fig. 11.

From Figs 10 and 11, reasonable consistency between analytical predictions and the simulation data is observed. 
Table 2. Collected data from the literature for comparison in Figs 12 and 13

\begin{tabular}{|c|c|c|c|c|}
\hline Relation & Study & Extracted data & Calculated data & Note \\
\hline $\begin{array}{l}k \text { against } \mathrm{Fr} \\
\alpha \text { against } \mathrm{Fr}\end{array}$ & $\begin{array}{l}\text { Scheidl et al. }(2013) \\
\text { Cui et al. }(2015) \\
\text { Hübl et al. }(2009) \\
\text { Hong et al. }(2015) \\
\text { Scotton }(1996) \\
\text { Scheidl et al. }(2013) \\
\text { Cui et al. }(2015) \\
\text { Bugnion et al. }(2012) \\
\text { Hübl et al. }(2009) \\
\text { Hong et al. }(2015)\end{array}$ & $\begin{array}{l}k, \mathrm{Fr} \\
p, \rho_{0}, u_{0}, h_{0}, \theta \\
k, \mathrm{Fr} \\
p, \rho_{0}, u_{0}, h_{0}, \theta=5 \cdot 1^{\circ} \text { from Cui et al. }(2005) \\
\alpha, \mathrm{Fr} \\
\alpha, \mathrm{Fr} \\
p, \rho_{0}, u_{0}, h_{0}, \theta \\
\alpha, u_{0}, h_{0}, \theta \\
\alpha, \mathrm{Fr} \\
p, \rho_{0}, u_{0}, h_{0}, \theta=5 \cdot 1^{\circ} \text { from Cui et al. }(2005)\end{array}$ & $\begin{array}{l}\text { N/A } \\
k, \mathrm{Fr} \\
\text { N/A } \\
k, \mathrm{Fr} \\
\text { N/A } \\
\text { N/A } \\
\alpha, \mathrm{Fr} \\
\mathrm{Fr} \\
\text { N/A } \\
\alpha, \mathrm{Fr}\end{array}$ & $\begin{array}{l}\text { Small-scale tests } \\
\text { Small-scale tests } \\
\text { Field events } \\
\text { Field events } \\
\text { Small-scale tests } \\
\text { Small-scale tests } \\
\text { Small-scale tests } \\
\text { Large-scale tests } \\
\text { Field events } \\
\text { Field events }\end{array}$ \\
\hline
\end{tabular}

The differences between the analytical predictions and the simulation results for groups I and II in both figures are relatively more obvious compared with that for group III. One reason for this may be the artificially generated debris flows in groups I and II at small Froude number. It is known that small-scale tests normally generate flows with a Froude number higher than 2 (Scheidl et al., 2013). The initial prismatic debris samples in both group I and group II are assigned with variable initial flow velocities to extend the range of Froude number to small values, but the flows in both groups are assumed to have a fixed initial height. In reality and in the present authors' simulations, the initial height may change (typically decrease) when the flow approaches the barrier. Consequently, the calculated $k$ based on the current simulations (i.e. filled circle and triangle symbols in Fig. 10) will be larger (due to using a smaller normaliser) than the analytical predictions (i.e. solid and dashed curves in Fig. 10).

In the small Froude number regime, the simulated $\alpha$ (i.e. filled circle and triangle symbols in Fig. 11) is also slightly higher than the analytical solutions (i.e. solid and dashed curves in Fig. 11). This may be due to the influence of interparticle friction and particle-wall friction in conjunction with the high solid fraction in groups I and II, which can quickly and significantly decrease the overall flow velocity. Indeed, the experimental results by Song et al. (2017) show that the velocity reduction of debris mixtures with higher solid fractions tends to be faster than that with lower solid fractions, which is related to the effect of grain contacts. As a result, the normalised $\alpha$ obtained from the simulations will be larger than the analytical predictions where a fixed incoming velocity is used for normalisation.

\section{Validation by experiment and field data reported in the literature}

The proposed analytical model is further validated by experimental data and real observations of debris flows in conjunction with the previous CFD-DEM simulation results. Table 2 summarises the collected data from the literature for the validation. In view of the heterogeneity of data with different natures, two bounding analytical solutions ('U' for upper and ' $L$ ' for lower) are presented and compared in Figs 12 and 13 along with the data. In addition, a design analytical solution ('D' for design) is also suggested based on parameters derived from the CFD-DEM simulations.

The rationale on parameter selection in plotting the analytical solutions in Figs 12 and 13 is as follows.

(a) $\beta$. In plotting analytical solutions $\mathrm{U}$ and $\mathrm{L}$, the present authors have chosen $\beta=1$. Indeed, their numerical simulations on debris mixtures across various flow regimes indicate a rather stable value for $\beta$ around 1 , with a maximum variation of $3 \cdot 1 \%$.

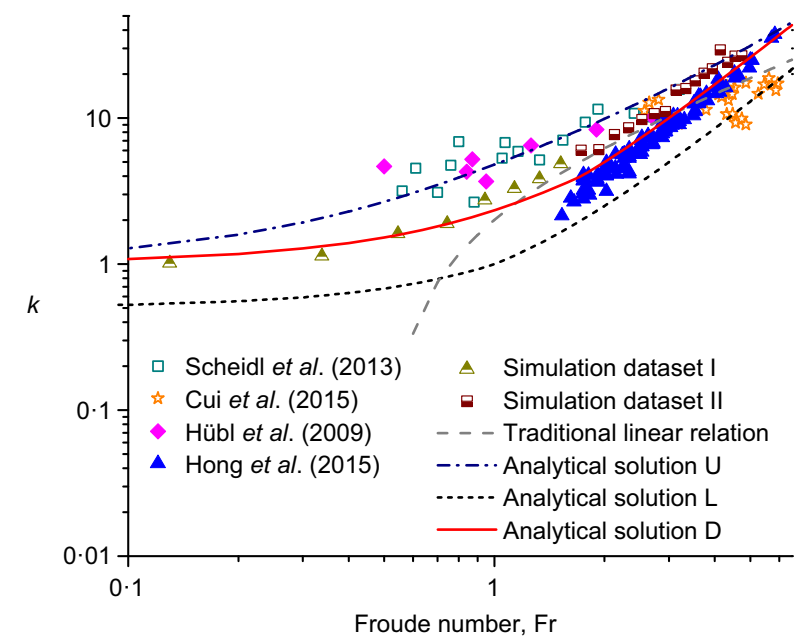

Fig. 12. The relation between the hydrostatic coefficient $k$ and Froude number $\mathrm{Fr}$, including data from small-scale experiments denoted by empty symbols (Scheidl et al., 2013; Cui et al., 2015), data from real debris flows denoted by solid symbols (Hübl et al., 2009; Hong et al., 2015), CFD-DEM simulation results and analytical solutions

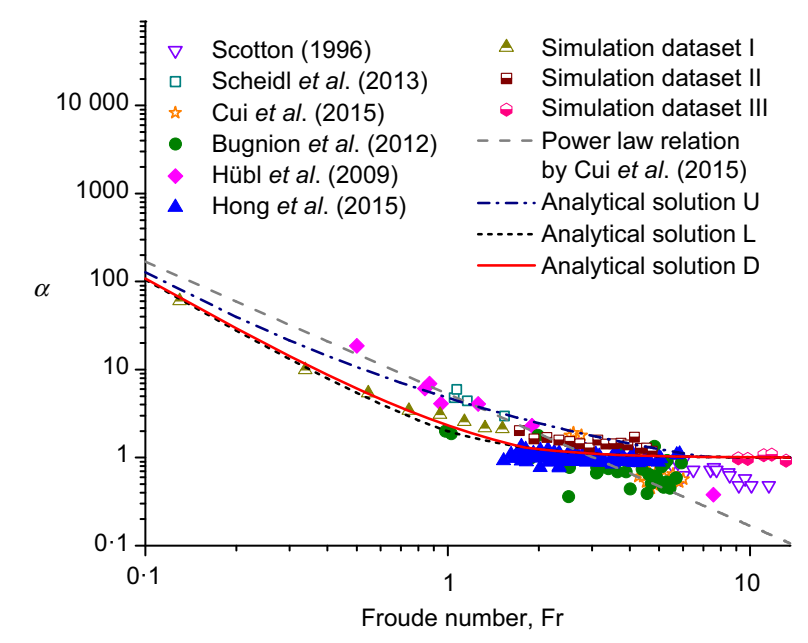

Fig. 13. The relation between the hydrodynamic coefficient $\alpha$ and Froude number $\mathrm{Fr}$, including data from small-scale experiments denoted by empty symbols (Scotton, 1996; Scheidl et al., 2013; Cui et al., 2015), data from large-scale tests and real debris flows denoted by solid symbols (Bugnion et al., 2012; Hübl et al., 2009; Hong et al., 2015), CFD-DEM simulation results and analytical solutions

(b) $\theta$ and $\kappa$. In plotting the analytical solution U, a slope angle $\theta=0^{\circ}$ and a pressure coefficient $\kappa=0 \cdot 15$ have been adopted. The analytical solution L in Figs 12 and 13 
adopts a slope angle $\theta=60^{\circ}$ and a pressure coefficient $\kappa=5 \cdot 0$. The slope angle from $0^{\circ}$ to $60^{\circ}$ refers to normal cases of debris flow (Takahashi, 2014; Nandi \& Shakoor, 2017). The pressure coefficient range is close to the typical value for dry, granular flows (i.e. $0 \cdot 2-5 \cdot 0$ recommended by Hungr (1995)). Instead of using $0 \cdot 2$ for the dry, granular flows, a smaller pressure coefficient $0 \cdot 15$ has been adopted in Figs 12 and 13 to cover more data of debris mixtures. A combination of $\theta=0^{\circ}$ and $\kappa=0 \cdot 15$ offers the largest coefficients $k$ and $\alpha$ within the considered range of $\theta$ and $\kappa$, which is therefore used for plotting the analytical solution $\mathrm{U}$ as an upper bound, and a combination of $\theta=60^{\circ}$ and $\kappa=5.0$ is adopted for analytical solution $\mathrm{L}$ as the lower bound.

(c) Fr. The Froude number of real debris flows is normally smaller than 2 (Hübl et al., 2009). The density ratio and pressure coefficient for analytical solution $\mathrm{D}$ are the average value from ten simulation cases with a Froude number smaller than 2 (eight cases in group I and two cases in group II), which are $\beta=0.997$ and $\kappa=1.873$, respectively. According to equations (21) and (22), when the density ratio, pressure coefficient and Froude number are all fixed, the ratio of the maximum run-up height to the height of incoming flow $h_{1} / h_{0}$ stays constant. A smaller slope angle leads to a larger $k$ but does not affect $\alpha$. Hence, the smallest slope angle $0^{\circ}$ is adopted in the analytical solution $\mathrm{D}$ for conservative reasons.

Figure 12 in terms of $k$ shows a good performance of the analytical solutions $U$ and $L$ in bounding the majority of data from a wide range of debris flows, including those from simulations, small-scale experiments and real observations. The few data above the analytical solution $U$ may correspond to cases with pressure coefficients smaller than $0 \cdot 15$, indicating very low tangential stresses of the flows compared with their normal stresses. The data below the analytical solution $\mathrm{L}$ are consistent with highly converging flows, whose pressure coefficients may be larger than $5 \cdot 0$.

In terms of $\alpha$, Fig. 13 demonstrates that the analytical solution U largely serves as a reasonable upper bound, whereas the analytical solution $\mathrm{L}$ is not necessarily lower than some of collected data, especially when the Froude number is larger than 2 . This may be due to the overestimated $h_{1} / h_{0}$ in the calculation of $\alpha$ using equation (22). The adopted $h_{1} / h_{0}$ in the analytical solution $\mathrm{L}$ has been based on energy conservation, as it is more conservative compared with the one from mass and momentum conservation. Notably, the study has adopted parameters based on CFD-DEM simulations and with relatively conservative consideration. This is due to a lack of experimental measurements on the changes of flow density $(\beta)$ and stresses along different directions $(\kappa)$ during the impact. More accurate (and/or economical) analytical models need rigorous calibration of parameters based on carefully designed experimental data and wellinstrumented observations of real debris flows.

\section{Further comparison with empirical predictions}

In addition to the experimental and field data reported in the literature, predictions of $k$ and $\alpha$ by empirical relations are plotted as gray dashed curves in Figs 12 and 13. The relation between the empirical hydrostatic coefficient $k$ and the Froude number Fr in Fig. 12 has been developed based on data from small-scale experiments and field-scale observations of debris flows (Hübl et al., 2009; Proske et al., 2011; Scheidl et al., 2013), which presents a linear relation as follows

$$
k=a \mathrm{Fr}+b
$$

A power law correlation has been established between the empirical hydrodynamic coefficient $\alpha$ and the Froude number Fr as follows (Hübl et al., 2003, 2009; Proske et al., 2011; Scheidl et al., 2013; Cui et al., 2015)

$$
\alpha=c \mathrm{Fr}^{-d}
$$

where $a, c$ and $d$ are positive constants and $b$ is a coefficient which may be zero (Scheidl et al., 2013) or non-zero (Hübl et al., 2009).

The present authors' finding of non-linear dependence of $k$ on the Froude number Fr deviates apparently from the conventional linear fitting curve $(k=4 \cdot 18 \mathrm{Fr}-2 \cdot 18)$ in Fig. 12 (note the double-log plot changes the line into a curve). The present authors' non-linear analytical solutions (U, L and D) in Fig. 12 all show an increasing rate of increase in $k$ with Fr, which shares a similar characteristic with the impact model considering drag force by Vagnon \& Segalini (2016). In particular, $k$ begins to grow significantly when Froude number goes beyond 1 , the boundary between subcritical and super-critical flows. Evidently, flow inertia is more dominant over gravitational force in a super-critical flow $(\mathrm{Fr}>1)$, leading to increasingly less weight of the static component in the overall pressure compared to the dynamic part. Fig. 12 demonstrates that the non-linear analytical solution D agrees better with the collected data when compared with the linear fitting curve, especially when the Froude number is smaller than one.

The empical power law relation plotted in Fig. 13 has been based on the study by Cui et al. (2015) with an expression of $\alpha=5 \cdot 3 \mathrm{Fr}^{-1 \cdot 5}$, whose trend differs essentially from the present authors' new analytical solution, especially at the tail part when Froude number is high. The traditional power law relation for $\alpha$ and Fr predicts that $\alpha=0$ at an infinite Froude number, indicating an apparently unrealistic zero impact pressure at very large flow velocity. In contrast, the present authors' new relation reasonably indicates a positive impact pressure at infinitely large flow velocity, which agrees with the relation proposed by Wang et al. (2018). It is interesting to observe a stagnant stage when Fr is high, which corresponds well to the stage of the sharp increase in $k$ (see Fig. 12). Indeed, after the flow inertia dominates over the gravitational force, $k$ needs to grow rapidly due to less weight of the static component, while $\alpha$ decreases due to the increasing weight of the dynamic component. The reduction of $\alpha$ in Fig. 13 is constrained by 1 , as indicated by equation (23).

\section{CONCLUSIONS AND DISCUSSION}

A new analytical model has been proposed to predict the peak impact pressure of debris flow on a rigid barrier. It is expressed in an additive form of a static part and a dynamic part in calculating the peak impact pressure. The dynamic pressure is derived according to the Bernoulli equation to consider the dynamic impact of an incoming flow. Accounting for the accumulation of flow on the barrier, the static pressure is calculated by the maximum run-up height obtained from a conservative solution of two sets of governing equations (mass and momentum conservation and energy conservation). Conventional pure hydraulic models have been re-evaluated, correlated and linked with the key parameters of the new model and Froude number, Fr. A simple reciprocal relationship has been established for conventional hydrostatic and hydrodynamic coefficients $k$ and $\alpha$, revealing an interesting competing mechanism of the hydrostatic and hydrodynamic components in understanding the impact of debris flow.

The new proposal has further been validated by collective data from small-scale experimental tests and real-scale 
observations, as well as coupled CFD-DEM simulations of debris flow impact on a rigid barrier. A coupled CFD-DEM modelling has been conducted to simulate a debris mixture consisting of solid particles and fluid, to assess realistically the impact of a two-phase debris flow. The numerical tool also enables convenient simulation of debris flows with a wide range of Froude numbers, covering those obtained in laboratory tests and field observations. It is demonstrated that the predictions by the new model offer reasonable consistency with all three datasets (numerical, experimental and field). In particular, suitable model parameters are calibrated from the numerical simulations and are used to provide upper and lower bounding solutions to cover the full-range of datasets available. A design solution is further offered for possible practical design.

The study offers a physically based consideration of debris flow impact, notwithstanding some oversimplified assumptions. Real debris flows are considerably more complicated than what the present authors have assumed. For example, turbulence and secondary surges in a debris flow may notably affect its impact behaviour. The liquid and solid phases in a debris mixture may have non-negligible separations, which may affect the flows. Further improvements can be achieved by considering heterogeneous incoming flow (e.g. more coarse solids and boulders) rather than a homogeneous flow. Detail governing the interaction mechanisms during the impact, such as the cushion effect of the dead zone, can also be included in future considerations. To render the model useful, it is also essential to develop practical ways to calibrate the model parameters accurately from experiments and field observations for a specific debris site.

\section{ACKNOWLEDGEMENTS}

The research reported in this study was financially supported by the National Natural Science Foundation of China (project \#11972030) and the University Grants Council of Hong Kong (RGC/GRF project \#16205418, TBRS project \#T22-603/15N and CRF project C6012-15G).

\section{APPENDIX 1. DERIVATION FROM EQUATION (5) \\ TO EQUATION (6)}

From the mass conservation

$$
\begin{aligned}
& \rho_{0} h_{0} u_{0}+\rho_{0} h_{0} u_{1}=\rho_{1} h_{1} u_{1} \\
& \rho_{0} h_{0} u_{0}=u_{1}\left(\rho_{1} h_{1}-\rho_{0} h_{0}\right) \\
& u_{1}=\frac{\rho_{0} h_{0} u_{0}}{\rho_{1} h_{1}-\rho_{0} h_{0}}=\frac{h_{0} u_{0}}{\beta h_{1}-h_{0}}
\end{aligned}
$$

From the momentum conservation

$$
\begin{aligned}
& \frac{1}{2} \kappa\left(\rho_{0} h_{0}^{2}-\rho_{1} h_{1}^{2}\right) \boldsymbol{g} \cos \theta=\rho_{1} h_{1} u_{1}^{2}-\rho_{0} h_{0}\left(u_{0}+u_{1}\right)^{2} \\
& \frac{\kappa \boldsymbol{g} \cos \theta}{2}\left(h_{0}^{2}-\beta h_{1}^{2}\right)=\beta h_{1} u_{1}^{2}-h_{0}\left(u_{0}^{2}+2 u_{0} u_{1}+u_{1}^{2}\right) \\
& \frac{\kappa \boldsymbol{g} \cos \theta}{2}\left(h_{0}^{2}-\beta h_{1}^{2}\right)=u_{1}^{2}\left(\beta h_{1}-h_{0}\right)-2 u_{1} u_{0} h_{0}-u_{0}^{2} h_{0}
\end{aligned}
$$

Substituting equation (29) into equation (32), the following are obtained

$$
\begin{aligned}
& \frac{\kappa \boldsymbol{g} \cos \theta}{2}\left(h_{0}^{2}-\beta h_{1}^{2}\right)=\left(\frac{h_{0} u_{0}}{\beta h_{1}-h_{0}}\right)^{2} \\
& \left(\beta h_{1}-h_{0}\right)-2\left(\frac{h_{0} u_{0}}{\beta h_{1}-h_{0}}\right) u_{0} h_{0}-u_{0}^{2} h_{0}
\end{aligned}
$$

$$
\begin{aligned}
& \frac{\kappa \boldsymbol{g} \cos \theta}{2}\left(h_{0}^{2}-\beta h_{1}^{2}\right)=\frac{u_{0}^{2} h_{0}^{2}}{\beta h_{1}-h_{0}}-2\left(\frac{u_{0}^{2} h_{0}^{2}}{\beta h_{1}-h_{0}}\right)-u_{0}^{2} h_{0} \\
& \frac{\kappa \boldsymbol{g} \cos \theta}{2}\left(h_{0}^{2}-\beta h_{1}^{2}\right)=\frac{u_{0}^{2} h_{0}^{2}}{h_{0}-\beta h_{1}}-u_{0}^{2} h_{0} \\
& \frac{\kappa g \cos \theta}{2}\left(1-\beta \frac{h_{1}^{2}}{h_{0}^{2}}\right)=\frac{u_{0}^{2}}{h_{0}-\beta h_{1}}-\frac{u_{0}^{2}}{h_{0}} \\
& \frac{\kappa g \cos \theta}{2}\left(1-\beta \frac{h_{1}^{2}}{h_{0}^{2}}\right)=\frac{u_{0}^{2} h_{0}}{\left(h_{0}-\beta h_{1}\right) h_{0}} \\
& -\frac{u_{0}^{2}\left(h_{0}-\beta h_{1}\right)}{\left(h_{0}-\beta h_{1}\right) h_{0}}=\frac{\beta u_{0}^{2} h_{1}}{\left(h_{0}-\beta h_{1}\right) h_{0}} \\
& \frac{\kappa \boldsymbol{g} \cos \theta}{2}\left(1-\beta \frac{h_{1}^{2}}{h_{0}^{2}}\right)=\frac{\beta u_{0}^{2} h_{1}}{\left[h_{0}-\beta h_{1}\left(h_{0} / h_{0}\right)\right] h_{0}}=\frac{\beta u_{0}^{2} h_{1}}{\left[1-\beta\left(h_{1} / h_{0}\right)\right] h_{0}^{2}} \\
& \left(1-\beta \frac{h_{1}^{2}}{h_{0}^{2}}\right)\left(1-\beta \frac{h_{1}}{h_{0}}\right)=\frac{2 \beta u_{0}^{2} h_{1}}{\kappa h_{0}^{2} g \cos \theta} \\
& =2 \beta \frac{u_{0}^{2}}{\kappa g h h_{0} \cos \theta} \frac{h_{1}}{h_{0}}=2 \beta \frac{\mathrm{Fr}^{2}}{\kappa} \frac{h_{1}}{h_{0}} \\
& {\left[\beta\left(\frac{h_{1}}{h_{0}}\right)^{2}-1\right]\left(\beta \frac{h_{1}}{h_{0}}-1\right)=2 \beta \frac{\mathrm{Fr}^{2}}{\kappa} \frac{h_{1}}{h_{0}}} \\
& \beta^{2}\left(\frac{h_{1}}{h_{0}}\right)^{3}-\beta\left(\frac{h_{1}}{h_{0}}\right)^{2}-\beta \frac{h_{1}}{h_{0}}+1=2 \beta \frac{\mathrm{Fr}^{2}}{\kappa} \frac{h_{1}}{h_{0}} \\
& \beta^{2}\left(\frac{h_{1}}{h_{0}}\right)^{3}-\beta\left(\frac{h_{1}}{h_{0}}\right)^{2}-\beta\left(1+\frac{2 \mathrm{Fr}^{2}}{\kappa}\right) \frac{h_{1}}{h_{0}}+1=0 \\
& \beta\left(\frac{h_{1}}{h_{0}}\right)^{3}-\left(\frac{h_{1}}{h_{0}}\right)^{2}-\left(1+\frac{2 \mathrm{Fr}^{2}}{\kappa}\right) \frac{h_{1}}{h_{0}}+\frac{1}{\beta}=0
\end{aligned}
$$

\section{APPENDIX 2. DEPENDENCY BETWEEN} EMPIRICAL COEFFICIENTS $k$ AND $\alpha$

From conventional hydraulic models (equations (15) and (16))

$$
\begin{gathered}
\rho_{0} g h_{0}=\frac{p}{k} \\
\rho_{0} u_{0}^{2}=\frac{p}{\alpha}
\end{gathered}
$$

Substituting equations (44) and (45) into equation (3)

$$
p=\alpha_{1} \frac{p}{k}+0 \cdot 5 \frac{p}{\alpha}
$$

which can be simplified as

$$
\frac{\alpha_{1}}{k}+\frac{0 \cdot 5}{\alpha}=1
$$

From the definition of Froude number equation (7), the following is obtained

$$
\mathrm{Fr}^{2}=\frac{u_{0}^{2}}{\boldsymbol{g} h_{0} \cos \theta}=\frac{\rho_{0} u_{0}^{2}}{\rho_{0} \boldsymbol{g} h_{0}} \frac{1}{\cos \theta}
$$

Substituting equations (44) and (45) into equation (48)

$$
\mathrm{Fr}^{2}=\frac{p / \alpha}{p / k} \frac{1}{\cos \theta}=\frac{k}{\alpha} \frac{1}{\cos \theta}
$$


Substituting the $h_{1} / h_{0}$ from energy conservation (equation (14)) into equation (4)

$$
\alpha_{1}=\frac{h_{1}}{h_{0}} \cos \theta=\left(1+0 \cdot 5 \mathrm{Fr}^{2}\right) \cos \theta
$$

Substituting equation (49) into equation (50)

$$
\alpha_{1}=\left(1+0 \cdot 5 \frac{k}{\alpha} \frac{1}{\cos \theta}\right) \cos \theta=\cos \theta+0 \cdot 5 \frac{k}{\alpha}
$$

Substituting equation (51) into equation (47), a reciprocal relation between $k$ and $\alpha$ can be obtained

$$
\frac{\cos \theta}{k}+\frac{1}{\alpha}=1
$$

If the $h_{1} / h_{0}$ from mass and momentum conservation is applied in equation (4), the following expression of $\alpha_{1}$ is obtained, instead of equation (50)

$$
\alpha_{1}=\frac{h_{1}}{h_{0}} \cos \theta=\left(2 \sqrt[3]{r} \cos \zeta+\frac{1}{3 \beta}\right) \cos \theta
$$

where $r$ and $\zeta$ are correlated with pressure coefficient $\kappa$, density ratio $\beta$ and Froude number Fr according to equations (9)-(12).

Substituting equation (53) into equation (47)

$$
\left(2 \sqrt[3]{r} \cos \zeta+\frac{1}{3 \beta}\right) \frac{\cos \theta}{k}+\frac{0 \cdot 5}{\alpha}=1
$$

where $r$ and $\zeta$ can be denoted with the following expressions by combining equations (9)-(12) and equation (49)

$$
\begin{aligned}
& r=\left(\frac{1}{3 \beta}+\frac{2 k}{3 \beta \alpha \kappa \cos \theta}+\frac{1}{9 \beta^{2}}\right)^{3 / 2} \\
& \zeta=\frac{1}{3} \arccos \left[\frac{-\left(2 / 3 \beta^{2}\right)+\left(2 k / 3 \beta^{2} \alpha \kappa \cos \theta\right)+\left(2 / 27 \beta^{3}\right)}{2\left[(1 / 3 \beta)+(2 k / 3 \beta \alpha \kappa \cos \theta)+\left(1 / 9 \beta^{2}\right)\right]^{3 / 2}}\right]
\end{aligned}
$$

Based on equations (54)-(56), $k$ and $\alpha$ can be determined from each other, giving $\kappa, \beta$ and $\theta$.

\section{NOTATION}

$a, b$ coefficients in the empirical correlation between $k$ and $\mathrm{Fr}$

$c, d$ coefficients in the empirical correlation between $\alpha$ and $\mathrm{Fr}$

$d_{\mathrm{p}}$ particle diameter

$e, q, r, \zeta$ constants in Cardano's solution of a cubic equation

Fr Froude number

g gravitational acceleration

$h$ initial flow height

$h_{0}$ height of incoming debris flow

$h_{1}$ maximum run-up height of debris flow

$k$ empirical hydrostatic coefficient

$L_{\mathrm{c}}$ channel length

$l$ initial length of debris sample

$m$ mass of considered flow point in energy conservation

$n, \kappa_{\mathrm{c}}, \tau_{\mathrm{c}}$ flow index, consistency and yield stress of debris fluid

$p$ peak impact pressure

$p_{\mathrm{s}} \quad$ static pressure

$u$ initial flow velocity

$u_{0}$ velocity of incoming debris flow

$u_{1} \quad$ velocity of reflected debris flow

$x, y$ coordinate axes

$\alpha$ empirical hydrodynamic coefficient

$\alpha_{1} \quad$ static coefficient

$\beta$ density ratio

$\theta$ slope angle

$\kappa$ pressure coefficient

$\rho_{0} \quad$ density of incoming debris flow

$\rho_{1}$ density of reflected debris flow

$\sigma_{1} \quad$ normal stress of debris flow

$\sigma_{3}$ tangential stress of debris flow

\section{REFERENCES}

Anderson, T. B. \& Jackson, R. (1967). Fluid mechanical description of fluidized beds. Equations of motion. Ind. Engng Chem. Fundam. 6, No. 4, 527-539.

Arattano, M. \& Franzi, L. (2003). On the evaluation of debris flows dynamics by means of mathematical models. Nat. Hazard Earth Sys. Sci. 3, No. 6, 539-544.

Armanini, A. (1997). On the dynamic impact of debris flows. In Recent developments on debris flows (eds A. Armanini and M. Michiue), pp. 208-226. Berlin/Heidelberg, Germany: Springer.

Armanini, A., Larcher, M. \& Odorizzi, M. (2011). Dynamic impact of a debris flow front against a vertical wall. In Proceedings of the 5 th international conference on debris-flow hazards mitigation: mechanics, prediction and assessment (eds R. Genevois, D. L. Hamilton and A. Prestininzi), pp. 1041-1049. Rome, Italy: Casa Editrice Universita La Sapienza.

Bugnion, L., McArdell, B. W., Bartelt, P. \& Wendeler, C. (2012). Measurements of hillslope debris flow impact pressure on obstacles. Landslides 9, No. 2, 179-187.

Byerlee, J. (1978). Friction of rocks. In Rock friction and earthquake prediction (eds J. D. Byerlee and M. Wyss), pp. 615-626. Basel, Switzerland: Birkhäuser.

Cardano, G. (1545). Artis magnae, sive de regulis algebraicis, liber unus. Milan, Italy: Joh. Petreius (in Latin).

Ceccato, F., Simonini, P., di Prisco, C. \& Redaelli, I. (2017). The effect of the front inclination on the impact forces transmitted by granular flows to rigid structures. In Workshop on world landslide forum (eds M. Mikos, B. Tiwari, Y. Yin and K. Sassa), pp. 593-599. Cham, Switzerland: Springer.

Choi, C. E., Au-Yeung, S. C. H., Ng, C. W. W. \& Song, D. (2015). Flume investigation of landslide granular debris and water run up mechanisms. Géotechnique Lett. 5, No. 1, 28-32, https://doi. org/10.1680/geolett.14.00080.

Coussot, P. \& Meunier, M. (1996). Recognition, classification and mechanical description of debris flows. Earth Sci. Rev. 40, No. 3-4, 209-227.

Cui, P., Chen, X. Q., Wang, Y. Y., Hu, K. H. \& Li, Y. (2005) Jiang-jia ravine debris flows in south-western China. In Debris-flow hazard and related phenomena (eds M. Jakob and O. Hungr), pp. 565-594. Berlin, Germany: Springer.

Cui, P., Zeng, C. \& Lei, Y. (2015). Experimental analysis on the impact force of viscous debris flow. Earth Surf. Processes Landforms 10, No. 12, 1644-1655.

Erhard, P., Etling, D., Muller, U., Riedel, U., Sreenivasan, K. R. \& Warnatz, J. (2010). Prandtl-essentials of fluid mechanics. New York, NY, USA: Springer Science \& Business Media.

Goniva, C., Kloss, C., Hager, A. \& Pirker, S. (2010). An open source CFD-DEM perspective. Proceedings of OpenFOAM workshop, Göteborg, Sweden, pp. 1-10.

Hübl, J., Holzinger, G. \& Wehrmann, H. (2003). Entwicklung von Grundlagen zur Dimensionierung kronenoffener Bauwerke für die Geschiebebewirtschaftung in Wildbächen: Klassifikation von Wildbachsperren, WLS Report 50, Im Auftrag des BMLFUW VC 7a (unveröffentlicht). Wien, Austria: Institut für Alpine Naturgefahren, Universität für Bodenkultur (in German).

Hübl, J., Suda, J., Proske, D., Kaitna, R. \& Scheidl, C. (2009). Debris flow impact estimation. In Proceedings of the 11th international symposium on water management and hydraulic engineering (eds C. Popovska and M. Jovanovski), pp. 1-5. Skopje, North Macedonia: University of Ss Cyril and Methodius.

Hong, Y., Wang, J. P., Li, D. Q., Cao, Z. J., Ng, C. W. W. \& Cui, P. (2015). Statistical and probabilistic analyses of impact pressure and discharge of debris flow from 139 events during 1961 and 2000 at Jiangjia Ravine, China. Particuology 187, 122-134.

Hu, K., Wei, F. \& Li, Y. (2011). Real-time measurement and preliminary analysis of debris-flow impact force at Jiangjia Ravine, China. Earth Surf. Processes Landforms 36, No. 9, $1268-1278$.

Huang, X. \& Garcia, M. H. (1998). A Herschel-Bulkley model for mud flow down a slope. J. Fluid Mech. 374, 305-333.

Hungr, O. (1995). A model for the runout analysis of rapid flow slides, debris flows, and avalanches. Can. Geotech. J. 32, No. 4, $610-623$. 
Hungr, O., Morgan, G. C. \& Kellerhals, R. (1984). Quantitative analysis of debris torrent hazards for design of remedial measures. Can. Geotech. J. 21, No. 4, 663-677.

Hurley, R. C., Hall, S. A., Andrade, J. E. \& Wright, J. (2016). Quantifying interparticle forces and heterogeneity in 3D granular materials. Phys. Rev. Lett. 117, No. 9, 098005.

Iverson, R. M. \& Denlinger, R. P. (2001). Flow of variably fluidized granular masses across three-dimensional terrain: 1. Coulomb mixture theory. J. Geophys. Res. Solid Earth 106, No. B1, 537-552.

Iverson, R. M., Logan, M., LaHusen, R. G. \& Berti, M. (2010). The perfect debris flow? Aggregated results from 28 large-scale experiments. J. Geophys. Res. Solid Earth 115, No. F3, article F03005.

Iverson, R. M., George, D. L. \& Logan, M. (2016). Debris flow runup on vertical barriers and adverse slopes. J. Geophys. Res. Solid Earth 121, No. 12, 2333-2357.

Jóhannesson, T., Gauer, P., Issler, P., Lied, K. \& Hákonardóttir, K. M. (2009). The design of avalanche protection dams: recent practical and theoretical developments, Tech Report. Brussels, Belgium: European Commission.

Kherkheulidze, I. (1969). Estimation of basic characteristics of mudflows ('sel'). In Floods and their computation: proceedings of the Leningrad symposium, August 1967, vol. 2, pp. 940-948. Wallingford, UK: International Association of Scientific Hydrology Publication.

Kloss, C. \& Goniva, C. (2010). LIGGGHTS: a new open source discrete element simulation software. Proceedings of the fifth international conference on discrete element methods, London, UK, pp. 25-26.

Kwan, J. S. H. (2012). Supplementary technical guidance on design of rigid debris-resisting barriers, GEO Report, No. 270. Hong Kong SAR: Geotechnical Engineering Office, HKSAR.

Li, X. Y. \& Zhao, J. D. (2018a). Dam-break of mixtures consisting of non-Newtonian liquids and granular particles. Powder Technol. 338, 493-505.

Li, X. Y. \& Zhao, J. D. (2018b). A unified CFD-DEM approach for modeling of debris flow impacts on flexible barriers. Int. J. Numer. Analyt. Methods Geomech. 42, No. 14, 1643-1670.

Lichtenhahn, C. (1973). Die Berechnung von Sperren in Beton und Eisenbeton. In Kolloquium über Wildbach-Sperren, Mitteilungen der Forstlichen Bundesanstalt Wien, pp. 91-127 (in German).

Lo, D. O. K. (2000). Review of natural terrain landslide debrisresisting barrier design. Hong Kong SAR: Geotechnical Engineering Office, Civil Engineering Department.

Nandi, A. \& Shakoor, A. (2017). Predicting debris flow initiation zone using statistical and rock kinematic analyses, a case study from West Prong Little Pigeon River, TN. Proceedings of the 3rd North American symposium on landslides (NASL), Roanoke, VA, USA, pp. 912-920.
Ng, C. W. W., Choi, C. E., Liu, L. H. D., Wang, Y., Song, D. \& Yang, N. (2017). Influence of particle size on the mechanism of dry granular run-up on a rigid barrier. Géotechnique Lett. 7, No. 1, 79-89, https://doi.org/10.1680/jgele.16.00159.

Proske, D., Suda, J. \& Hübl, J. (2011). Debris flow impact estimation for breakers. Georisk 5, No. 2, 143-155.

Rahman, M. A.\& Konagai, K. (2017). A hands-on approach to estimate debris flow velocity for rational mitigation of debris hazard. Can. Geotech. J. 55, No. 7, 941-955.

Scheidl, C., Chiari, M., Kaitna, R., Müllegger, M., Krawtschuk, A., Zimmermann, T. \& Proske, D. (2013). Analysing debris-flow impact models, based on a small scale modelling approach. Surv. Geophys. 34, No. 1, 121-140.

Scheidl, C., McArdell, B. W. \& Rickenmann, D. (2014). Debris-flow velocities and superelevation in a curved laboratory channel. Can. Geotech. J. 52, No. 3, 305-317.

Scotton, P. (1996). Dynamic impact of debris flows: experimental study. Trento, Italy: Università di Trento. Dipartimento di Ingegneria Civile e Ambientale.

Shan, T. \& Zhao, J. D. (2014). A coupled CFD-DEM analysis of granular flow impacting on water reservoir. Acta Mechanica 225, No. 8, 2449-2470.

Song, D., Ng, C. W. W., Choi, C. E., Zhou, G. G. D., Kwan, J. S. H. \& Koo, R. C. H. (2017). Influence of debris flow solid fraction on rigid barrier impact. Can. Geotech. J. 54, No. 10, 1421-1434.

Takahashi, T. (2014). Debris flow: mechanics, prediction and countermeasures. Leiden, the Netherlands: CRC Press/Balkema.

Tsuji, Y., Tanaka, T. \& Ishida, T. (1992). Lagrangian numerical simulation of plug flow of cohesionless particles in a horizontal pipe. Powder Technol. 71, No. 3, 239-250.

Tsuji, Y., Kawaguchi, T. \& Tanaka, T. (1993). Discrete particle simulation of two-dimensional fluidized bed. Powder Technol. 77, No. 1, 79-87.

Vagnon, F. \& Segalini, A. (2016). Debris flow impact estimation on a rigid barrier. Nat. Hazard Earth Sys. Sci. 16, No. 7, 1691-1697.

Viccione, G., Ferlisi, S. \& Marra, E. (2015). A numerical investigation of the interaction between debris flows and defense barriers. Advances in environmental and geological science and engineering, proceedings of the 8th international conference on environmental and geological science and engineering (EG'15) (eds A. Bulucea, G. Viccione and C. Guarnaccia), pp. 332-342. Athens, Greece: WSEAS Press.

Wang, Y., Liu, X., Yao, C., Li, Y., Liu, S. \& Zhang, X. (2018). Finite release of debris flows around round and square piers. J. Hydraul. Engng 144, No. 12, 06018015.

Weller, H. G., Tabor, G., Jasak, H. \& Fureby, C. (1998). A tensorial approach to computational continuum mechanics using object-oriented techniques. Comput. Phys. 12, No. 6, 620-631.

Zhao, J. \& Shan, T. (2013). Numerical modeling of fluid-particle interaction in granular media. Theor. App. Mech. Lett. 3, No. 2, 021007. 